\title{
A NEW CHARACTERIZATION OF SOME ALTERNATING AND SYMMETRIC GROUPS
}

\author{
AMIR KHOSRAVI and BEHROOZ KHOSRAVI
}

Received 5 February 2002

\begin{abstract}
We suppose that $p=2^{\alpha} 3^{\beta}+1$, where $\alpha \geq 1, \beta \geq 0$, and $p \geq 7$ is a prime number. Then we prove that the simple groups $A_{n}$, where $n=p, p+1$, or $p+2$, and finite groups $S_{n}$, where $n=p, p+1$, are also uniquely determined by their order components. As corollaries of these results, the validity of a conjecture of J. G. Thompson and a conjecture of Shi and Bi (1990) both on $A_{n}$, where $n=p, p+1$, or $p+2$, is obtained. Also we generalize these conjectures for the groups $S_{n}$, where $n=p, p+1$.
\end{abstract}

2000 Mathematics Subject Classification: 20D05, 20D60, 20 D08.

1. Introduction. Let $G$ be a finite group. We denote by $\pi(G)$ the set of all prime divisors of $|G|$. We construct the prime graph of $G$ as follows. The prime $\operatorname{graph} \Gamma(G)$ of a group $G$ is the graph whose vertex set is $\pi(G)$, and two distinct primes $p$ and $q$ are joined by an edge (we write $p \sim q$ ) if and only if $G$ contains an element of order $p q$. Let $t(G)$ be the number of connected components of $\Gamma(G)$ and let $\pi_{1}, \pi_{2}, \ldots, \pi_{t(G)}$ be the connected components of $\Gamma(G)$. If $2 \in \pi(G)$, then we always suppose that $2 \in \pi_{1}$.

Now $|G|$ can be expressed as a product of coprime positive integers $m_{i}$, $i=1,2, \ldots, t(G)$, where $\pi\left(m_{i}\right)=\pi_{i}$. These integers are called the order components of $G$. The set of order components of $G$ will be denoted by OC $(G)$. Also we call $m_{2}, \ldots, m_{t(G)}$ the odd-order components of $G$. The order components of non-abelian simple groups having at least three prime graph components are obtained by Chen [7, Tables 1, 2, 3]. Similarly, the order components of nonabelian simple groups with two-order components can be obtained by using the tables in [18, 28].

The following groups are uniquely determined by their order components: Suzuki-Ree groups [6], Sporadic simple groups [4], $\operatorname{PSL}_{2}(q)$ [7], $E_{8}(q)$ [2], $G_{2}(q)$, where $q \equiv 0(\bmod 3)$ [3], $F_{4}(q)$, where $q$ is even [15], $\operatorname{PSL}_{3}(q)$, where $q$ is an odd prime power [14], $\operatorname{PSL}_{3}(q)$, where $q=2^{n}$ [13], $\operatorname{PSU}_{3}(q)$, where $q>5$ [16], and $A_{p}$, where $p$ and $p-2$ are primes [12].

It was proved by Oyama [20] that a finite group which has the same table of characters as an alternating group $A_{n}$ is isomorphic to $A_{n}$. It was also proved by Koike [17] that a finite group which has the isomorphic subgroup-lattice as an alternating group $A_{n}$ is isomorphic to $A_{n}$. 
Let $\pi_{e}(G)$ denote the set of orders of elements in $G$. Shi and Bi [27] proved that if $\pi_{e}(G)=\pi_{e}\left(A_{n}\right)$ and $|G|=\left|A_{n}\right|$, then $G \cong A_{n}$. Iranmanesh and Alavi [12] proved that if $p$ and $p-2$ are primes and $\mathrm{OC}(G)=\mathrm{OC}\left(A_{p}\right)$, then $G \cong A_{p}$. Praeger and Shi [21] and Shi and Bi [26] proved that $A_{8}, A_{9}, A_{11}, A_{13}, S_{7}$, and $S_{8}$ are characterizable by their element orders. Also recently, Kondrat'ev and Mazurov [19] and Zavarnitsin [29] proved that if $\pi_{e}(G)=\pi_{e}\left(A_{n}\right)$, where $n=$ $s, s+1, s+2$ and $s$ is a prime number, then $G \cong A_{n}$.

Now we prove the following theorems.

THEOREM 1.1. Let $p=2^{\alpha} 3^{\beta}+1$, where $\alpha \geq 1, \beta \geq 0$, and $p \geq 7$ is a prime number. Let $M=A_{n}$, where $n=p, p+1, p+2$. Then $\mathrm{OC}(G)=\mathrm{OC}(M)$ if and only if $G \cong M$.

THEOREM 1.2. Let $p=2^{\alpha} 3^{\beta}+1$, where $\alpha \geq 1, \beta \geq 0$, and $p \geq 7$ is a prime number. Let $M=S_{n}$, where $n=p, p+1$. Then $\mathrm{OC}(G)=\mathrm{OC}(M)$ if and only if $G \cong M$.

In this paper, all groups are finite and by simple groups we mean non-abelian simple groups. All further unexplained notations are standard and we refer, for example, to [10]. Also frequently we use the results of Williams [28] and Kondrat'ev [18] about the prime graph of simple groups.

\section{Preliminary results}

REMARK 2.1. Let $N$ be a normal subgroup of $G$ and $p \sim q$ in $\Gamma(G / N)$. Then $p \sim q$ in $\Gamma(G)$. In fact if $x N \in G / N$ has order $p q$, then there is a power of $x$ which has order $p q$.

DEFINITION 2.2 (see [11]). A finite group $G$ is called a 2-Frobenius group if it has a normal series $1 \unlhd H \unlhd K \unlhd G$, where $K$ and $G / H$ are Frobenius groups with kernels $H$ and $K / H$, respectively.

LEMMA 2.3 (see [28, Theorem A]). If $G$ is a finite group with its prime graph having more than one component, then $G$ is one of the following groups:

(a) a Frobenius or 2-Frobenius group;

(b) a simple group;

(c) an extension of a $\pi_{1}$-group by a simple group;

(d) an extension of a simple group by a $\pi_{1}$-solvable group;

(e) an extension of a $\pi_{1}$-group by a simple group by a $\pi_{1}$-group.

LEMMA 2.4 (see [28, Lemma 3]). If $G$ is a finite group with more than one prime graph component and has a normal series $1 \unlhd H \unlhd K \unlhd G$ such that $H$ and $G / K$ are $\pi_{1}$-groups and $K / H$ is a simple group, then $H$ is a nilpotent group.

The next lemma follows from [1, Theorem 2].

LEMMA 2.5. Let $G$ be a Frobenius group of even order and let $H, K$ be Frobenius complement and Frobenius kernel of $G$, respectively. Then $t(\Gamma(G))=2$, 
and the prime graph components of $G$ are $\pi(H), \pi(K)$ and $G$ has one of the following structures:

(a) $2 \in \pi(K)$ and all Sylow subgroups of $H$ are cyclic;

(b) $2 \in \pi(H), K$ is an abelian group, $H$ is a solvable group, the Sylow subgroups of odd order of $H$ are cyclic groups, and the 2-Sylow subgroups of $H$ are cyclic or generalized quaternion groups;

(c) $2 \in \pi(H), K$ is an abelian group, and there exists $H_{0} \leq H$ such that $\left|H: H_{0}\right| \leq 2, H_{0}=Z \times \operatorname{SL}(2,5),(|Z|, 2.3 .5)=1$, and the Sylow subgroups of $Z$ are cyclic.

The next lemma follows from [1, Theorem 2] and Lemma 2.4.

LEMMA 2.6. Let $G$ be a 2-Frobenius group of even order. Then $t(\Gamma(G))=2$ and $G$ has a normal series $1 \unlhd H \unlhd K \unlhd G$ such that

(a) $\pi_{1}=\pi(G / K) \cup \pi(H)$ and $\pi(K / H)=\pi_{2}$;

(b) $G / K$ and $K / H$ are cyclic, $|G / K|$ divides $|\operatorname{Aut}(K / H)|,(|G / K|,|K / H|)=1$, and $|G / K|<|K / H|$;

(c) $H$ is nilpotent and $G$ is a solvable group.

LEMMA 2.7 (see [8, Lemma 8]). Let $G$ be a finite group with $t(\Gamma(G)) \geq 2$ and let $N$ be a normal subgroup of $G$. If $N$ is a $\pi_{i}$-group for some prime graph component of $G$ and $m_{1}, m_{2}, \ldots, m_{r}$ are some order components of $G$ but not a $\pi_{i}$-number, then $m_{1} m_{2} \cdots m_{r}$ is a divisor of $|N|-1$.

The next lemma follows from [5, Lemma 1.4].

LEMMA 2.8. Suppose that $G$ and $M$ are two finite groups satisfying $t(\Gamma(M)) \geq$ 2, $N(G)=N(M)$, where $N(G)=\{n \mid G$ has a conjugacy class of size $n\}$, and $Z(G)=1$. Then $|G|=|M|$.

LEMMA 2.9 (see [5, Lemma 1.5]). Let $G_{1}$ and $G_{2}$ be finite groups satisfying $\left|G_{1}\right|=\left|G_{2}\right|$ and $N\left(G_{1}\right)=N\left(G_{2}\right)$. Then $t\left(\Gamma\left(G_{1}\right)\right)=t\left(\Gamma\left(G_{2}\right)\right)$ and $\mathrm{OC}\left(G_{1}\right)=$ $\mathrm{OC}\left(G_{2}\right)$.

LEMMA 2.10. Let $G$ be a finite group and let $M$ be a non-abelian finite group with $t(M)=2$ satisfying $\mathrm{OC}(G)=\mathrm{OC}(M)$.

(1) Let $|M|=m_{1} m_{2}$, OC $(M)=\left\{m_{1}, m_{2}\right\}$, and $\pi\left(m_{i}\right)=\pi_{i}$ for $i=1$, 2 . Then $|G|=m_{1} m_{2}$ and one of the following holds:

(a) $G$ is a Frobenius or 2-Frobenius group;

(b) $G$ has a normal series $1 \unlhd H \unlhd K \unlhd G$ such that $G / K$ is a $\pi_{1}$-group, $H$ is a nilpotent $\pi_{1}$-group, and $K / H$ is a non-abelian simple group. Moreover, $\mathrm{OC}(K / H)=\left\{m_{1}^{\prime}, m_{2}^{\prime}, \ldots, m_{s}^{\prime}, m_{2}\right\},|K / H|=m_{1}^{\prime} m_{2}^{\prime} \cdots m_{s}^{\prime} m_{2}$, and $m_{1}^{\prime} m_{2}^{\prime} \cdots m_{s}^{\prime} \mid m_{1}$, where $\pi\left(m_{j}^{\prime}\right)=\pi_{j}^{\prime}, 1 \leq j \leq s$.

(2) In case (b), $|G / K||| \operatorname{Out}(K / H) \mid$.

Proof. The proof of (1) follows from the above lemmas. Since $t(G) \geq 2$, we have $t(G / H) \geq 2$. Otherwise $t(G / H)=1$, so that $t(G)=1$. Since $H$ is a $\pi_{i}$-group, 
TABLE 2.1

\begin{tabular}{|c|c|}
\hline$p$ & Finite simple $C_{p p}$ groups \\
\hline 2 & $\begin{array}{l}A_{5}, A_{6} \text {; } \\
L_{2}(q), \text { where } q \text { is a Fermat prime, a Mersenne prime, or } q=2^{n}, n \geq 3 \text {, } \\
L_{3}\left(2^{2}\right), S z\left(2^{2 n+1}\right), n \geq 1\end{array}$ \\
\hline 3 & $\begin{array}{l}A_{5}, A_{6} ; \\
L_{2}(q), \text { where } q=2^{3}, 3^{n+1} \text {, or } 2.3^{n} \pm 1 \text { which is a prime, } n \geq 1, L_{3}\left(2^{2}\right)\end{array}$ \\
\hline 5 & $\begin{array}{l}A_{5}, A_{6}, A_{7} ; M_{11}, M_{22} ; \\
L_{2}(q), \text { where } q=7^{2}, 5^{n} \text {, or } 2.5^{n} \pm 1 \text { which is a prime, } n \geq 1, L_{3}\left(2^{2}\right), \\
S_{4}(q), q=3,7, U_{4}(3) ; \\
S z(q), q=2^{3}, 2^{5}\end{array}$ \\
\hline 7 & $\begin{array}{l}A_{7}, A_{8}, A_{9} ; M_{22}, J_{1}, J_{2}, H S \\
L_{2}(q), q=2^{3}, 7^{n}, \text { or } 2.7^{n}-1 \text { which is a prime, } n \geq 1, L_{3}\left(2^{2}\right), S_{6}(2), \\
O_{8}^{+}(2), G_{2}(q), q=3,19 ; \\
U_{3}(q), q=3,5,19 ; U_{4}(3), U_{6}(2), S z\left(2^{3}\right)\end{array}$ \\
\hline 13 & $\begin{array}{l}A_{13}, A_{14}, A_{15} ; S u z, F i_{22} \text {; } \\
L_{2}(q), q=3^{3}, 5^{2}, 13^{n} \text {, or } 2.13^{n}-1 \text { which is a prime, } n \geq 1, L_{3}(3) \text {, } \\
L_{4}(3), O_{7}(3), S_{4}(5), S_{6}(3), O_{8}^{+}(3), G_{2}(q), q=2^{3}, 3 ; \\
F_{4}(2), U_{3}(q), q=2^{2}, 23, S z\left(2^{3}\right),{ }^{3} D_{4}(2),{ }^{2} E_{6}(2),{ }^{2} F_{4}(2)^{\prime}\end{array}$ \\
\hline 17 & $\begin{array}{l}A_{17}, A_{18}, A_{19} ; J_{3}, H e, F i_{23}, F i_{24}^{\prime} \\
L_{2}(q), q=2^{4}, 17^{n}, 2.17^{n} \pm 1 \text { which is a prime, } n \geq 1, S_{4}(4), S_{8}(2), \\
F_{4}(2), O_{8}^{-}(2), O_{10}^{-}(2),{ }^{2} E_{6}(2)\end{array}$ \\
\hline 19 & $\begin{array}{l}A_{19}, A_{20}, A_{21} ; \\
J_{1}, J_{3}, O^{\prime} N, T h, H N ; L_{2}(q), q=19^{n}, 2.19^{n}-1 \text { which is a prime, } n \geq 1 \text {, } \\
L_{3}(7), U_{3}\left(2^{3}\right), R\left(3^{3}\right),{ }^{2} E_{6}(2)\end{array}$ \\
\hline 37 & $\begin{array}{l}A_{37}, A_{38}, A_{39} ; J_{4}, L y ; \\
L_{2}(q), q=37^{n}, 2.37^{n}-1 \text { which is a prime, } n \geq 1 \\
U_{3}(11), R\left(3^{3}\right),{ }^{2} F_{4}\left(2^{3}\right)\end{array}$ \\
\hline 73 & $\begin{array}{l}A_{73}, A_{74}, A_{75} ; \\
L_{2}(q), q=73^{n}, 2.73^{n}-1 \text { which is a prime, } n \geq 1, L_{3}\left(2^{3}\right), S_{6}\left(2^{3}\right), \\
G_{2}(q), q=2^{3}, 3^{2} ; \\
F_{4}(3), E_{6}(2), E_{7}(2), U_{3}\left(3^{2}\right),{ }^{3} D_{4}(3)\end{array}$ \\
\hline 109 & $\begin{array}{l}A_{109}, A_{110}, A_{111} \\
L_{2}(q), q=109^{n}, 2.109^{n}-1 \text { which is a prime, } n \geq 1,{ }^{2} F_{4}\left(2^{3}\right)\end{array}$ \\
\hline $\begin{array}{l}p=2^{m}+1 \\
m=2^{s}\end{array}$ & $\begin{array}{l}A_{p}, A_{p+1}, A_{p+2} \\
L_{2}(q), q=2^{m}, p^{k}, 2 \cdot p^{k} \pm 1 \text { which is a prime, } s \geq k \geq 1, S_{a}\left(2^{b}\right), \\
a=2^{c+1} \text { and } b=2^{d}, c \geq 1, c+d=s, F_{4}\left(2^{e}\right), e \geq 1,4 e=2^{s}, \\
O_{2(m+1)}^{-}(2), s \geq 2, O_{a}^{-}\left(2^{b}\right), c \geq 2, c+d=s\end{array}$ \\
\hline Other & $A_{p}, A_{p+1}, A_{p+2} ; L_{2}(q), q=p^{k}, 2 \cdot p^{k}-1$ which is a prime, $k \geq 1$ \\
\hline
\end{tabular}

we arrive at a contradiction. Moreover, we have $Z(G / H)=1$. For any $x H \in G / H$ and $x H \notin K / H, x H$ induces an automorphism of $K / H$ and this automorphism 
is trivial if and only if $x H \in Z(G / H)$. Therefore, $G / K \leq \operatorname{Out}(K / H)$ and since $Z(G / H)=1$, (2) follows.

DEFINITION 2.11. A group $G$ is called a $C_{p p}$ group if the centralizers of its elements of order $p$ in $G$ are $p$-groups.

LEMMA 2.12 (see [9]). Let $p$ be a prime and $p=2^{\alpha} 3^{\beta}+1, \alpha \geq 0$ and $\beta \geq 0$. Then any finite simple $C_{p p}$ group is given by Table 2.1.

3. Characterization of some alternating and symmetric groups. In the sequel, we suppose that $p=2^{\alpha} 3^{\beta}+1$, where $\alpha \geq 1, \beta \geq 0$, and $p \geq 7$ is a prime number.

LEMMA 3.1. Let $G$ be a finite group and let $M$ be $A_{n}$, where $n=p, p+1$, or $p+2$, or $S_{n}$, where $n=p, p+1$. If $\mathrm{OC}(G)=\mathrm{OC}(M)$, then $G$ is neither a Frobenius group nor a 2-Frobenius group.

Proof. If $G$ is a Frobenius group, then by Lemma 2.5, OC $(G)=\{|H|,|K|\}$, where $K$ and $H$ are Frobenius kernel and Frobenius complement of $G$, respectively. Since $|H||| K \mid-1$, we have $|H|<|K|$. Therefore, $2 \nmid|H|$, and hence 2||$K \mid$. So, $|H|=p,|K|=|G| / p$. We claim that there exists a prime $p^{\prime}$ such that $3 n / 4<p^{\prime}$. Note that $p \leq n$, and hence $p^{\prime 2} \nmid\left|A_{n}\right|$. Let $\beta(n)$ be the number of prime numbers less than or equal to $n$. In fact, by [22, Theorem 2] we have

$$
\frac{n}{\log n-1 / 2}<\beta(n)<\frac{n}{\log n-3 / 2},
$$

where $n \geq 67$. Thus

$$
\beta(n)-\beta\left(\frac{3 n}{4}\right)>\frac{n}{\log n-1 / 2}-\frac{3 n / 4}{\log (3 n / 4)-3 / 2} .
$$

When $n \geq 405$, we get $\beta(n)-\beta(3 n / 4)>1$, and for $n<405$, we can immediately obtain the result by checking the table of prime numbers. Now let $P^{\prime}$ be the $p^{\prime}$-Sylow subgroup of $K$. Since $K$ is nilpotent, $P^{\prime} \triangleleft G$. Then $p \mid p^{\prime}-1$, by Lemma 2.7, which is a contradiction since $p^{\prime}<p$. Therefore, $G$ is not a Frobenius group.

Now let $G$ be a 2-Frobenius group. By Lemma 2.6, there is a normal series $1 \unlhd$ $H \unlhd K \unlhd G$ such that $|K / H|=p$ and $|G / K|<p$. So, $|H| \neq 1$ since $|G|=|G / K|$. $|K / H| \cdot|H|$. Since 2||$H \mid$, let $p^{\prime}$ be as above and let $P^{\prime}$ be the $p^{\prime}$-Sylow subgroup of $H$. Now, $p \mid p^{\prime}-1$, which is impossible. Hence, $G$ is not a 2-Frobenius group.

LEMMA 3.2. Let $G$ be a finite group and $M=A_{n}$, where $n=p, p+1$, or $p+2$, or $S_{n}$, where $n=p, p+1$. If $\mathrm{OC}(G)=\mathrm{OC}(M)$, then $G$ has a normal series $1 \unlhd H \unlhd K \unlhd G$ such that $H$ and $G / K$ are $\pi_{1}$-groups and $K / H$ is a simple group. Moreover, the odd-order component of $M$ is equal to an odd-order component of $K / H$. In particular, $t(\Gamma(K / H)) \geq 2$. Also $|G / H|$ divides $|\operatorname{Aut}(K / H)|$, and in fact $G / H \leq \operatorname{Aut}(K / H)$. 
Proof. The first part of the lemma follows from the above lemmas since the prime graph of $M$ has two prime graph components. For primes $p$ and $q$, if $K / H$ has an element of order $p q$, then $G$ has one. Hence, by the definition of prime graph component, the odd-order component of $G$ must be an odd-order component of $K / H$. Since $K / H \triangleleft G / H$ and $C_{G / H}(K / H)=1$, we have

$$
G / H=\frac{N_{G / H}(K / H)}{C_{G / H}(K / H)} \cong T, \quad T \leq \operatorname{Aut}(K / H) .
$$

THEOREM 3.3. Let $p=2^{\alpha} 3^{\beta}+1$, where $\alpha \geq 1, \beta \geq 0$, and $p \geq 7$ is a prime number. Let $M=A_{n}$, where $n=p, p+1, p+2$. Then $\mathrm{OC}(G)=\mathrm{OC}(M)$ if and only if $G \cong M$.

Proof. By Lemma 3.2, $G$ has a normal series $1 \unlhd H \unlhd K \unlhd G$ such that $\pi(H) \bigcup \pi(G / K) \subset \pi_{1}, K / H$ is a non-abelian simple group, $t(\Gamma(K / H)) \geq 2$, and the odd-order component of $M$ is an odd-order component of $K / H$. Therefore, $K / H$ is a finite simple $C_{p p}$ group. Now using Table 2.1, we consider each possibility of $K / H$ separately.

In the sequel, we frequently use the results of [28, Table I] and [18, Tables 2, 3].

STEP 1. Let $p=7,13,17,19,37,73$, or 109 .

Since the proofs of these cases are similar, we state only one of them, say $p=13$. Using Table 2.1, we have

(1) $K / H \cong S u z$ or $F i_{22}$. It is a contradiction since $3^{7}|| S u z \mid$ and $3^{9}|| F i_{22} \mid$ but $3^{7} \nmid\left|A_{n}\right|$, where $n=13,14,15$;

(2) $K / H \cong L_{2}(27), L_{2}(25), L_{3}(3), L_{4}(3), S z(8),{ }^{2} F_{4}(2)^{\prime}$, or $U_{3}(4)$. If $K / H \cong$ $L_{2}(27)$, then $|G| /|K / H|=|H| \cdot|G / K| \neq 1$. By Lemma 2.6, $|G / K||| \operatorname{Out}(K /$ $H) \mid=6$. So, $|H| \neq 1$. Let $P$ be the 5-Sylow subgroup of $H$. But since $H$ is nilpotent, $P \triangleleft G$. Hence, $13 \mid(|P|-1)$, which is a contradiction. Other cases are similar;

(3) $K / H \cong L_{2}\left(13^{r}\right)$ or $L_{2}\left(2.13^{r}-1\right)$, where $2.13^{r}-1$ is a prime, $r \geq 1$. Note that $13^{2} \nmid|G|$, hence $r=1$. So, $K / H \cong L_{2}(13)$ or $L_{2}(25)$, and we can proceed similar to (2);

(4) $K / H \cong O_{7}(3)$. It is a contradiction since $3^{9}|| O_{7}(3) \mid$ but $3^{9} \nmid\left|A_{n}\right|$;

(5) $K / H \cong S_{4}(5)$ or $S_{6}(3)$. It is a contradiction since $5^{4}|| S_{4}(5) \mid$ but $5^{4} \nmid\left|A_{n}\right|$. Also $3^{9}|| S_{6}(3) \mid$ but $3^{9} \nmid\left|A_{n}\right|$;

(6) $K / H \cong O_{8}^{+}(3)$. It is a contradiction since $3^{12}|| O_{8}^{+}(3) \mid$ but $3^{12} \nmid\left|A_{n}\right|$;

(7) $K / H \cong G_{2}$ (3) or $G_{2}(8)$. If $K / H \cong G_{2}(3)$, then we get a contradiction since for $n=13,14$ we have $3^{6}|| G_{2}(3) \mid$ but $3^{6} \nmid\left|A_{n}\right|$. For $n=15$, since $\left|\operatorname{Out}\left(G_{2}(3)\right)\right|=2$, we have $|H| \neq 1$. Now we proceed similar to (2). If $K / H \cong G_{2}(8)$, then we get a contradiction since $2^{18}|| G_{2}(8) \mid$ but $2^{18} \nmid\left|A_{n}\right| ;$

(8) $K / H \cong F_{4}(2)$. It is a contradiction since 17||$F_{4}(2) \mid$ but $17 \nmid\left|A_{n}\right|$;

(9) $K / H \cong U_{3}$ (23). It is a contradiction since 23||$U_{3}(23) \mid$ but $23 \nmid\left|A_{n}\right|$; 
(10) $K / H \cong{ }^{3} D_{4}(2)$ or ${ }^{2} E_{6}(2)$. It is a contradiction since $2^{12} \nmid\left|A_{n}\right|$. Also $19 \nmid$ $\left|A_{n}\right|$

(11) $K / H$ is an alternating group, namely $A_{13}, A_{14}$, or $A_{15}$.

First suppose that $n=13$. Since $|K / H| \leq\left|A_{13}\right|, K / H \cong A_{13}$. But $|G|=$ $\left|A_{13}\right|$, and hence $H=1$ and $K=G \cong A_{13}$. If $n=14$, then $K / H \cong A_{13}$ or $A_{14}$. But if $r \neq 6$, then $\operatorname{Aut}\left(A_{r}\right)=S_{r}$, and hence $\left|\operatorname{Out}\left(A_{r}\right)\right|=2$. If $K / H \cong$ $A_{13}$, then $|G / K| \mid 2$, and hence $|H| \neq 1$. Now we get a contradiction similar to (2). Therefore, $K / H \cong A_{14}$, and hence $G \cong A_{14}$. If $n=15$, we do similarly.

STEP 2. Let $p=2^{m}+1$, where $m=2^{s}$.

Using Table 2.1, we have

(i) $K / H \cong L_{2}\left(2^{m}\right)$. Note that for every $m$ we have $\left|L_{2}\left(2^{m}\right)\right||| G \mid$. Using Lemma 2.6, $|G / K||| \operatorname{Out}(K / H) \mid$. Also $\left|\operatorname{Out}\left(L_{2}\left(2^{m}\right)\right)\right|=m$. Hence, $|H| \neq$ 1 . Now let $p^{\prime}$ be a prime number less than $p$ such that

$$
p^{\prime} \| \frac{\left|A_{n}\right|}{m|K / H|}
$$

Let $P^{\prime}$ be the $p^{\prime}$-Sylow subgroup of $H$. Since $H$ is nilpotent, $P^{\prime} \triangleleft G$. Hence, $p \mid\left(\left|P^{\prime}\right|-1\right)$, which is a contradiction;

(ii) $K / H \cong L_{2}\left(p^{k}\right)$ or $L_{2}\left(2 p^{k} \pm 1\right)$, where $2 p^{k} \pm 1$ is a prime and $1 \leq k \leq s$. We know that $p \|\left|A_{n}\right|$, hence $k=1$. Now we proceed similar to (i);

(iii) $K / H \cong S_{a}\left(2^{b}\right)$, where $a=2^{c+1}$ and $b=2^{d}, c \geq 1, c+d=s$. Let $q=2^{b}$ and $f=2^{c}$, Then $p=q^{f}+1$ and we have

$$
\left|S_{a}\left(2^{b}\right)\right|=q^{f^{2}}\left(q^{f}-1\right)\left(q^{f}+1\right) \Pi_{i=1}^{f-1}\left(q^{i}-1\right)\left(q^{i}+1\right) .
$$

Each factor of the form $\left(q^{j} \pm 1\right)$ is less than or equal to $p$ and therefore divides $\left|A_{n}\right|$. Also $q^{f^{2}}=\left(2^{m}\right)^{f} \leq 2^{m 2} \leq 2^{2^{m}}$. Hence, $\left|S_{a}\left(2^{b}\right)\right||| A_{n} \mid$. But $\left|\operatorname{Out}\left(S_{a}\left(2^{b}\right)\right)\right|=b$. Then $|H| \neq 1$ and we can proceed similar to (i);

(iv) $K / H \cong F_{4}\left(2^{e}\right)$, where $e \geq 1,4 e=2^{s}$, or $O_{2(m+1)}^{-}$(2), where $s \geq 2$, or $O_{a}^{-}\left(2^{b}\right)$, where $c \geq 2, c+d=s$. Again this part is similar to (iii);

(v) $K / H \cong A_{p}, A_{p+1}, A_{p+2}$.

First suppose that $n=p$. Since $|K / H| \leq\left|A_{p}\right|, K / H \cong A_{p}$. But $|G|=$ $\left|A_{p}\right|$, and hence $H=1$ and $K=G \cong A_{p}$. If $n=p+1$, then $K / H \cong A_{p}$ or $A_{p+1}$. But if $r \neq 6$, then $\operatorname{Aut}\left(A_{r}\right)=S_{r}$, and hence $\left|\operatorname{Out}\left(A_{r}\right)\right|=2$. If $K / H \cong A_{p}$, then $|G / K| \mid 2$, and hence $|H| \neq 1$. Now we get a contradiction similar to (i). Therefore, $K / H \cong A_{p+1}$, and hence $G \cong A_{p+1}$. If $n=p+2$, we do similarly.

STEP 3. For other primes $p$, we have $K / H \cong A_{p}, A_{p+1}, A_{p+2} ; L_{2}(q)$, where $q=p^{k}, 2 p^{k}-1$ which is a prime, $k \geq 1$.

In fact the proof of this step is exactly similar to that of Step 2 and we omit it for convenience. 
THEOREM 3.4. If $G$ is a non-abelian finite group with connected prime graph, then $G$ is not characterizable with its order component.

Proof. Clearly, OC $(G)=\mathrm{OC}\left(\mathbb{Z}_{|G|}\right)$, but $G \supsetneqq \mathbb{Z}_{|G|}$.

COROLLARY 3.5. Every simple group with one component (see [28, Table I]) is not characterizable with this method.

THEOREM 3.6. Let $n$ be a positive integer. If there exist at least two nonisomorphic abelian groups of order $n$, then abelian groups of order $n$ are not characterizable with their order component.

Proof. The proof is obvious.

REMARK 3.7. It was a conjecture that every finite simple group $M$, where $\Gamma(M)$ is not connected, is characterizable with its order components. But the following example is a counterexample.

EXAMPLE 3.8. If $q$ is an odd-prime power and $n=2^{k} \geq 4$, then $\operatorname{OC}\left(S_{2 n}(q)\right)=$ OC $\left(O_{2 n+1}(q)\right)$, but obviously $S_{2 n}(q) \nRightarrow O_{2 n+1}(q)$.

THEOREM 3.9. Let $p=2^{\alpha} 3^{\beta}+1$, where $\alpha \geq 1, \beta \geq 0$, and $p \geq 7$ is a prime number. Let $M=S_{n}$, where $n=p, p+1$. Then $\mathrm{OC}(G)=\mathrm{OC}(M)$ if and only if $G \cong M$.

Proof. Similar to the proof of Theorem 3.3, since $G$ is a $C_{p p}$ group, we have $K / H \cong A_{n}$. Now using Lemma 3.2, we have

$$
A_{n} \leq \frac{G}{H} \leq \operatorname{Aut}\left(A_{n}\right)=S_{n}
$$

Therefore, $G / H \cong A_{n}$ or $\operatorname{Aut}\left(A_{n}\right)=S_{n}$. If $G / H \cong A_{n}$, then $|H|=2$ and $H \triangleleft G$. Hence, $H \subseteq Z(G)=1$, which is a contradiction. Therefore, $G / H \cong S_{n}$, and since $|G|=\left|S_{n}\right|$, we have $G \cong S_{n}$.

\section{Some related results}

REMARK 4.1. It is a well known conjecture of J. G. Thompson that if $G$ is a finite group with $Z(G)=1$ and $M$ is a non-abelian simple group satisfying $N(G)=N(M)$, then $G \cong M$.

We can generalize this conjecture for the groups under discussion by our characterization of these groups.

COROLlary 4.2. Let $G$ be a finite group with $Z(G)=1$ and let $M$ be $A_{p}$, $A_{p+1}, A_{p+2}, S_{p}$, or $S_{p+1}$. If $N(G)=N(M)$, then $G \cong M$.

Proof. By Lemmas 2.8 and 2.9, if $G$ and $M$ are two finite groups satisfying the conditions of Corollary 4.2, then $\mathrm{OC}(G)=\mathrm{OC}(M)$. So, Theorems 3.3 and 3.9 imply this corollary. 
REMARK 4.3. Shi and Bi in [26] put forward the following conjecture.

SHI's CONJECTURE. Let $G$ be a group and $M$ a finite simple group. Then $G \cong M$ if and only if

(i) $|G|=|M|$,

(ii) $\pi_{e}(G)=\pi_{e}(M)$, where $\pi_{e}(G)$ denotes the set of orders of elements in $G$.

This conjecture is valid for sporadic simple groups [24], groups of alternating type [27], and some simple groups of Lie type [23, 25, 26]. As a consequence of Theorems 3.3 and 3.9, we prove a generalization of this conjecture for the groups under discussion.

COROLlaRY 4.4. Let $G$ be a finite group and let $M$ be $A_{p}, A_{p+1}, A_{p+2}, S_{p}$, or $S_{p+1}$. If $|G|=|M|$ and $\pi_{e}(G)=\pi_{e}(M)$, then $G \cong M$.

Proof. By assumption, we must have $\mathrm{OC}(G)=\mathrm{OC}(M)$. Thus the corollary follows by Theorems 3.3 and 3.9.

\section{REFERENCES}

[1] G. Y. Chen, On structure of Frobenius groups and 2-Frobenius groups, J. Southwest China Normal Univ. 20 (1995), no. 5, 485-487.

[2] - A new characterization of $E_{8}(q)$, J. Southwest China Normal Univ. 21 (1996), no. 3, 215-217.

[3] _ A new characterization of $G_{2}(q),[q \equiv 0(\bmod 3)]$, J. Southwest China Normal Univ. (1996), 47-51.

[4] _ A new characterization of sporadic simple groups, Algebra Colloq. 3 (1996), no. 1, 49-58.

[5] _ On Thompson's conjecture, J. Algebra 185 (1996), no. 1, 184-193.

[6] A new characterization of Suzuki-Ree group, Sci. China Ser. A 40 (1997), no. 8, 807-812.

[7] - A new characterization of $\operatorname{PSL}_{2}$ (q), Southeast Asian Bull. Math. 22 (1998), no. 3, 257-263.

[8]___ Further reflections on Thompson's conjecture, J. Algebra 218 (1999), no. 1, 276-285.

[9] Z. M. Chen and W. J. Shi, On simple $C_{p p}$ groups, J. Southwest China Teachers Univ. (Nat. Sci) 18 (1993), no. 3, 249-256.

[10] J. H. Conway, R. T. Curtis, S. P. Norton, R. A. Parker, and R. A. Wilson, Atlas of Finite Groups, Oxford University Press, Oxford, 1985.

[11] K. W. Gruenberg and K. W. Roggenkamp, Decomposition of the augmentation ideal and of the relation modules of a finite group, Proc. London Math. Soc. (3) 31 (1975), no. 2, 149-166.

[12] A. Iranmanesh and S. H. Alavi, A new characterization of $A_{p}$ where $p$ and $p-2$ are primes, Korean J. Comput. Appl. Math. 8 (2001), no. 3, 665-673.

[13] A. Iranmanesh, S. H. Alavi, and B. Khosravi, A characterization of $\operatorname{PSL}(3, q)$ for $q=2^{m}$, Acta Math. Sin. (Engl. Ser.) 18 (2002), no. 3, 463-472.

[14] - A characterization of $\operatorname{PSL}(3, q)$ where $q$ is an odd prime power, J. Pure Appl. Algebra 170 (2002), no. 2-3, 243-254.

[15] A. Iranmanesh and B. Khosravi, A characterization of $F_{4}(q)$ where $q=2^{n}(n>1)$, Far East J. Math. Sci. 2 (2000), no. 6, 853-859. 
[16] A. Iranmanesh, B. Khosravi, and S. H. Alavi, A Characterization of $\operatorname{PSU}_{3}(q)$ for $q>5$, S. E. Bull. Math. 26 (2002), no. 1, 33-44.

[17] K. Koike, On some groups which are determined by their subgroup-lattices, Tokyo J. Math. 6 (1983), no. 2, 413-421.

[18] A. S. Kondrat'ev, Prime graph components of finite simple groups, Math. USSR-Sb. 67 (1990), no. 1, 235-247.

[19] A. S. Kondrat'ev and V. D. Mazurov, Recognition of alternating groups of prime degree from their element orders, Siberian Math. J. 41 (2000), no. 2, 294302.

[20] T. Oyama, On the groups with the same table of characters as alternating groups, Osaka J. Math. 1 (1964), no. 1, 91-101.

[21] C. E. Praeger and W. J. Shi, A characterization of some alternating and symmetric groups, Comm. Algebra 22 (1994), no. 5, 1507-1530.

[22] J. B. Rosser and L. Schoenfeld, Approximate formulas for some functions of prime numbers, Illinois J. Math. 6 (1962), 64-94.

[23] W. J. Shi, A new characterization of some simple groups of Lie type, Classical Groups and Related Topics (Beijing, 1987), Contemp. Math., vol. 82, American Mathematical Society, Rhode Island, 1989, pp. 171-180.

[24] _ A new characterization of the sporadic simple groups, Group Theory (Singapore, 1987), de Gruyter, Berlin, 1989, pp. 531-540.

[25] _ Pure quantitative characterization of finite simple groups. I, Progr. Natur. Sci. (English Ed.) 4 (1994), no. 3, 316-326.

[26] W. J. Shi and J. X. Bi, A characteristic property for each finite projective special linear group, Groups-Canberra 1989, Lecture Notes in Math., vol. 1456, Springer, Berlin, 1990, pp. 171-180.

[27] A new characterization of the alternating groups, Southeast Asian Bull. Math. 16 (1992), no. 1, 81-90.

[28] J. S. Williams, Prime graph components of finite groups, J. Algebra 69 (1981), no. $2,487-513$.

[29] A. V. Zavarnitsin, Recognition of alternating groups of degrees $r+1$ and $r+2$ for prime $r$ and of a group of degree 16 by the set of their element orders, Algebra i Logika 39 (2000), no. 6, 648-661 (Russian), translated in Algebra and Logic 39 (2000), no. 6, 370-377.

Amir Khosravi: Faculty of Mathematical Sciences and Computer Engineering, University for Teacher Education, 599 Taleghani Ave., Tehran 15614, Iran

Behrooz Khosravi: 241 Golnaz Street, Velenjak, Tehran 19847, Iran

E-mail address: khosravibbb@yahoo.com 


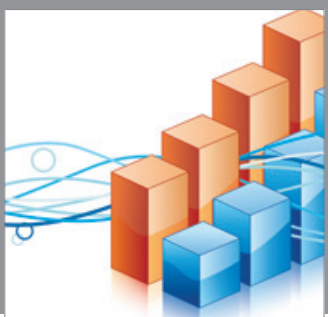

Advances in

Operations Research

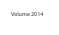

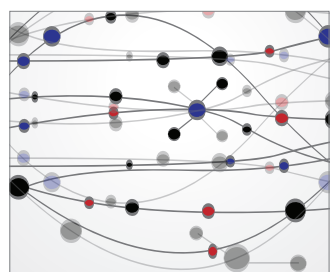

\section{The Scientific} World Journal
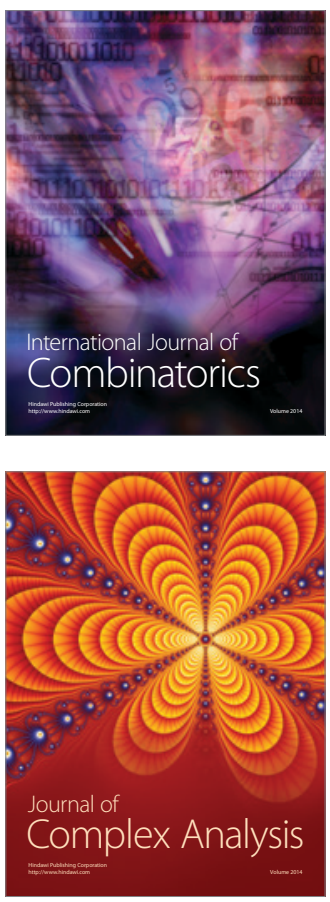

International Journal of

Mathematics and

Mathematical

Sciences
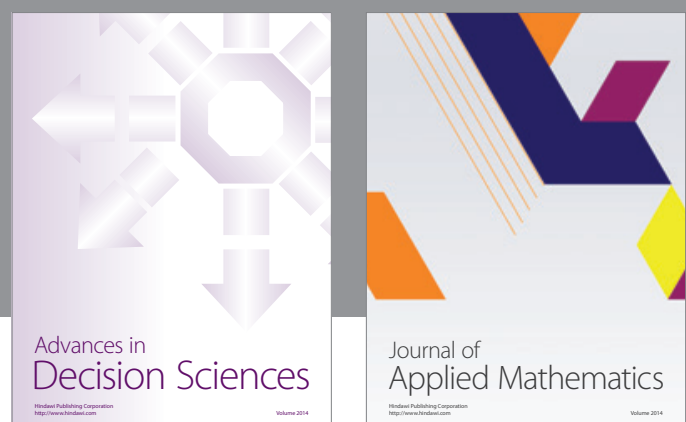

Journal of

Applied Mathematics
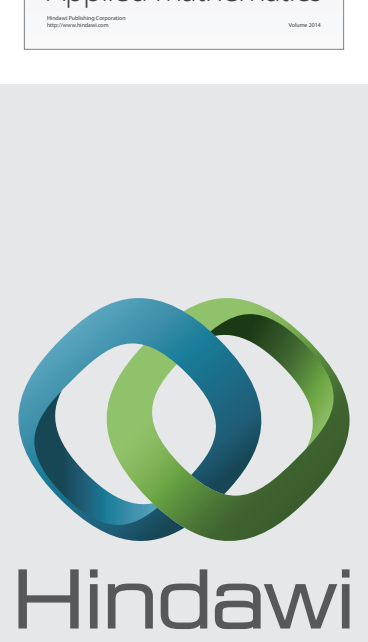

Submit your manuscripts at http://www.hindawi.com
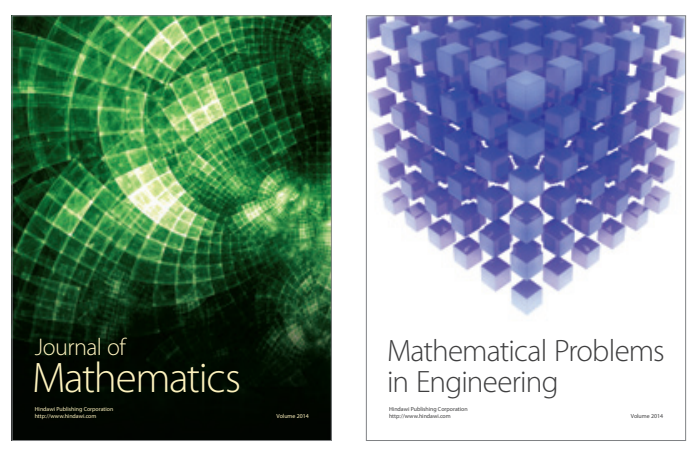

Mathematical Problems in Engineering
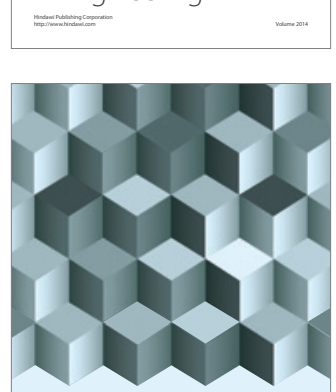

Journal of

Function Spaces
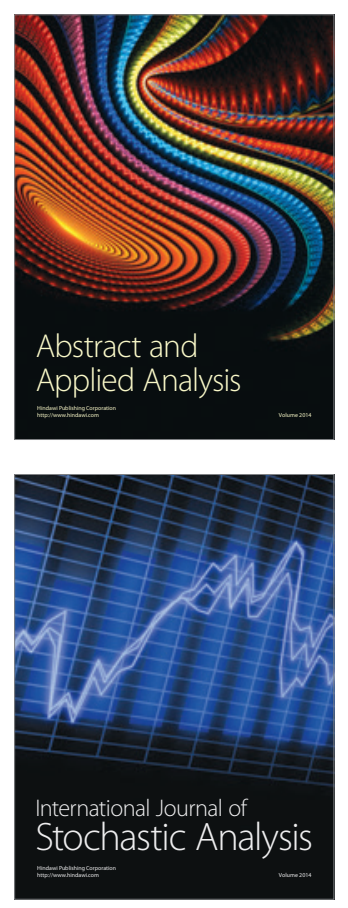

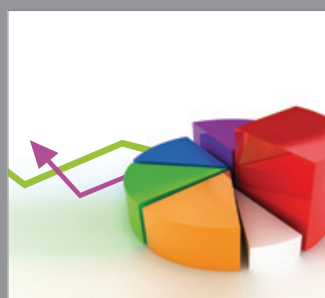

ournal of

Probability and Statistics

Promensencen
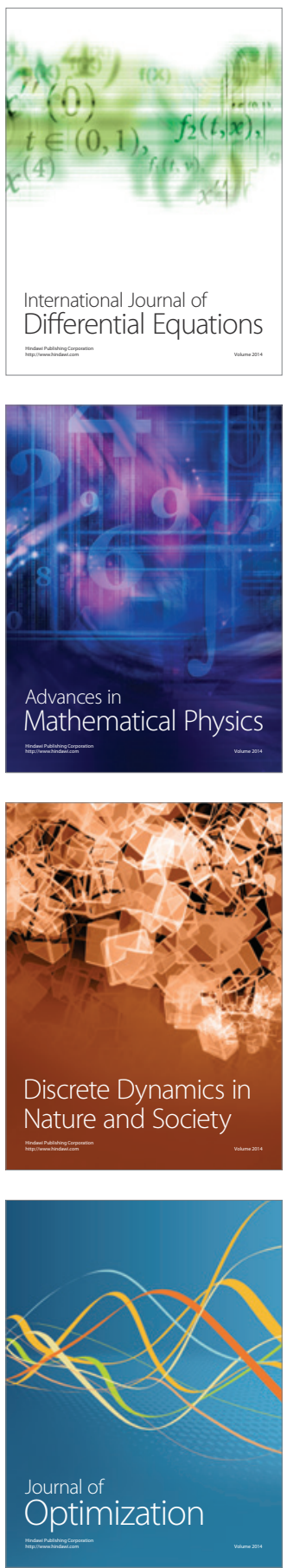